\title{
ON MEAN CONVERGENCE OF TRIGONOMETRIC INTERPOLANTS, AND THEIR UNIT CIRCLE ANALOGUES, FOR GENERAL ARRAYS
}

\author{
D.S. LUBINSKY
}

\begin{abstract}
Let $X$ be a triangular array of interpolation points in a compact subset of $[0,2 \pi]$. We obtain a necessary and sufficient condition for the existence of $p>0$ such that the associated trigonometric polynomials are convergent in $L_{p}$. We also examine Lagrange interpolation on the unit circle. The results are analogues of our earlier ones for Lagrange interpolation on a real interval.
\end{abstract}

\section{The Result}

In a recent paper [5], we showed how distribution functions and Loomis' Lemma can be used to obtain a simple necessary and sufficient condition for the existence of $p>0$ for which Lagrange interpolation polynomials converge in $L_{p}$. The interest in this lies in the simplicity of the proof and its general applicability. Most positive results on mean convergence of Lagrange interpolation are closely linked to zeros of orthogonal polynomials, and are somewhat technical - see [6], [8], [12], [13]. An extension to interpolation associated with weights on the real line was presented in [7], using decreasing rearrangements and an inequality of Hardy and Littlewood.

In this paper, we shall present an analogue for trigonometric interpolation and for interpolation on the unit circle. The main ideas are similar to those in [5], but there are some technical complications in the proofs. First, however, let us recall the result of [5]. Let $X$ be an array of interpolation points $X=\left\{x_{j n}\right\}_{1 \leq j \leq n, n \geq 1}$ in a compact set $K \subset \mathbb{R}$, with

$$
x_{n n}<x_{n-1, n}<\cdots<x_{2 n}<x_{1 n} .
$$

We denote by $L_{n}[\cdot]$ the associated Lagrange interpolation operator, so that for $f: K \rightarrow \mathbb{R}$, we have

$$
L_{n}[f](x)=\sum_{j=1}^{n} f\left(x_{j n}\right) \ell_{j n}(x),
$$

where the fundamental polynomials $\left\{\ell_{k n}\right\}_{k=1}^{n}$ satisfy

$$
\ell_{k n}\left(x_{j n}\right)=\delta_{j k} \text {. }
$$

We also let $\pi_{n}$ denote a polynomial of degree $n$ (without any specific normalisation) whose zeros are $\left\{x_{j n}\right\}_{j=1}^{n}$. Our result was:

\section{THEOREM 1}

Let $K \subset \mathbb{R}$ be compact, and let $v \in L_{q}(K)$ for some $q>0$. Let the array $X$ of

Date: 6 December 2001. 
interpolation points lie in $K$. The following are equivalent:

(I) There exists $p>0$ such that for every continuous $f: K \rightarrow \mathbb{R}$, we have

$$
\lim _{n \rightarrow \infty}\left\|\left(f-L_{n}[f]\right) v\right\|_{L_{p}(K)}=0 .
$$

(II) There exists $r>0$ such that

$$
\sup _{n \geq 1}\left\|\pi_{n} v\right\|_{L_{r}(K)}\left(\sum_{j=1}^{n} \frac{1}{\left|\pi_{n}^{\prime}\right|\left(x_{j n}\right)}\right)<\infty .
$$

The necessity of the condition in (II), was established by Ying Guang Shi [11]; the new feature of [5] was the more difficult sufficiency.

To formulate our trigonometric analogue, we need some notation. Let $\Theta=$ $\left\{\theta_{j n}\right\}_{0 \leq j \leq 2 n, n \geq 1}$ be an array of interpolation points in a compact set $K \subset[0,2 \pi]$, with

$$
\theta_{2 n, n}<\theta_{2 n-1, n}<\cdots<\theta_{2, n}<\theta_{1, n}<\theta_{0, n} .
$$

We denote by $\Upsilon_{n}[\cdot]$ the associated trigonometric interpolation operator, so that for $f: K \rightarrow \mathbb{R}$, we have

$$
\Upsilon_{n}[f](\theta)=\sum_{j=0}^{2 n} f\left(\theta_{j n}\right) \tau_{j n}(\theta),
$$

where the fundamental polynomials $\left\{\tau_{k n}\right\}_{k=0}^{2 n}$ are trigonometric polynomials of degree $2 n$ that satisfy

$$
\tau_{k n}\left(\theta_{j n}\right)=\delta_{j k}
$$

The formula for $\tau_{k n}$ is a little more complicated than its algebraic polynomial analogue. Let

$$
\omega_{n}(\theta):=\prod_{j=0}^{2 n}\left(2 \sin \left(\frac{\theta-\theta_{j n}}{2}\right)\right) .
$$

Then

$$
\tau_{j n}(\theta)=\frac{\omega_{n}(\theta)}{\omega_{n}^{\prime}\left(\theta_{j n}\right)\left(2 \sin \frac{\theta-\theta_{j n}}{2}\right)}
$$

[14, p. $174 \mathrm{ff}]$, [15]. (This is easily established with a little manipulation).

We let $\mathcal{C}(K)$ denote the class of all continuous functions $f: K \rightarrow \mathbb{C}$ with sup norm. If $K$ contains both 0 and $2 \pi$, we require in addition that $f$ is $2 \pi$-periodic, that is

$$
f(0)=f(2 \pi) .
$$

Our first result is:

\section{THEOREM 2}

Let $K \subset[0,2 \pi]$ be compact, and let $v \in L_{q}(K)$ for some $q>0$. Let the array $T$ of interpolation points lie in $K$. The following are equivalent:

(I) There exists $p>0$ such that for every $f \in \mathcal{C}(K)$, we have

$$
\lim _{n \rightarrow \infty}\left\|\left(f-\Upsilon_{n}[f]\right) v\right\|_{L_{p}(K)}=0 .
$$


(II) There exists $r>0$ such that

$$
\sup _{n \geq 1}\left\|\omega_{n} v\right\|_{L_{r}(K)}\left(\sum_{j=0}^{2 n} \frac{1}{\left|\omega_{n}^{\prime}\right|\left(\theta_{j n}\right)}\right)<\infty .
$$

Of course, trigonometric interpolation may be viewed as interpolation on the unit circle $\Gamma$ by Laurent polynomials

$$
\sum_{j=-n}^{n} c_{j} z^{j}
$$

While these are dense in $\mathcal{C}(K)$, interpreted as a space of functions defined on the unit circle, the ordinary polynomials are not: recall that if a sequence of polynomial converges uniformly on the unit circle, then the limit function must constitute the boundary values of a function analytic in the unit ball. So in formulating a result for polynomial interpolation on the unit circle, it is natural to consider Hardy spaces.

For functions $f$ analytic in the unit ball, and $0<p<\infty$, their Hardy space norm is

$$
\begin{aligned}
& \|\quad f\|_{H_{p}}:=\sup _{0 \leq r<1}\left(\frac{1}{2 \pi} \int_{0}^{2 \pi}\left|f\left(r e^{i \theta}\right)\right|^{p} d \theta\right)^{1 / p} \\
& =\lim _{r \rightarrow 1-}\left(\frac{1}{2 \pi} \int_{0}^{2 \pi}\left|f\left(r e^{i \theta}\right)\right|^{p} d \theta\right)^{1 / p} .
\end{aligned}
$$

If this norm is finite, we write $f \in H_{p}$. Then the non-tangential boundary values $f^{*}$ exist a.e. on the unit circle and

$$
\|f\|_{H_{p}}=\left(\frac{1}{2 \pi} \int_{0}^{2 \pi}\left|f^{*}\left(e^{i \theta}\right)\right|^{p} d \theta\right)^{1 / p}
$$

See [10] for further orientation. We also let $\bar{D}$ denote the closed unit ball, and $A(\bar{D})$ denote the space of functions continuous in $\bar{D}$ and analytic in the open unit ball, with uniform norm. It is well known that $A(\bar{D})$ coincides with the closure of the polynomials in the uniform norm on $\bar{D}$.

Let $Z=\left\{z_{j n}\right\}_{1 \leq j \leq n, n \geq 1}$ denote an array of interpolation points on the unit circle $\Gamma$, with $z_{1 n}, z_{2 n} \ldots z_{n n}$ distinct and let $L_{n}[\cdot]$ denote the associated Lagrange interpolation operator, so that for $f: \Gamma \rightarrow \mathbb{C}$, we have

$$
L_{n}[f](z)=\sum_{j=1}^{n} f\left(z_{j n}\right) \ell_{j n}(z),
$$

where the fundamental polynomials $\left\{\ell_{k n}\right\}_{k=1}^{n}$ satisfy

$$
\ell_{k n}\left(z_{j n}\right)=\delta_{j k} \text {. }
$$

We also let $\pi_{n}$ denote a polynomial of degree $n$ (without any specific normalisation) whose zeros are $\left\{z_{j n}\right\}_{j=1}^{n}$. Before stating our second result, we emphasize in the following simple proposition that limits of (interpolation) polynomials in $L_{p}(\Gamma)$ must lie in $H_{p}$ : 


\section{PROPOSITION}

Let $p>0$ and $f: \Gamma \rightarrow \mathbb{C}$ be measurable. Assume that

$$
\lim _{n \rightarrow \infty}\left\|f-L_{n}[f]\right\|_{L_{p}(\Gamma)}=0 .
$$

Then there exists $F \in H_{p}$ such that

$$
f=F^{*},
$$

that is, $f$ constitutes the non-tangential boundary values of some $F \in H_{p}$.

Our second result is:

\section{THEOREM 3}

Let $Z$ denote an array of interpolation points in $\Gamma$. The following are equivalent:

(I) There exists $p>0$ such that for $f \in A(\bar{D})$, we have

$$
\lim _{n \rightarrow \infty}\left\|f-L_{n}[f]\right\|_{H_{p}}=0 .
$$

(II) There exists $r>0$ such that

$$
\sup _{n \geq 1}\left\|\pi_{n}\right\|_{H_{r}}\left(\sum_{j=1}^{n} \frac{1}{\left|\pi_{n}^{\prime}\right|\left(z_{j n}\right)}\right)<\infty .
$$

Note that the convergence in $H_{p}$ norm also ensures that $\left\{L_{n}[f]\right\}_{n=1}^{\infty}$ converges uniformly to $f$ in compact subsets of the unit ball, even for $p<1$. Thus (4) also provides a sufficient condition on an array of interpolation points on the unit circle for locally uniform convergence of the interpolants inside the unit ball. As far as the author is aware, there are not that many arrays on the unit circle, for which this convergence is known, so (4) provides a relatively simple condition. Of course roots of unity are the archetypal example.

After this paper was accepted, the author noticed [2]. That paper gives a necessary and sufficient condition for convergence of Lagrange interpolation locally uniformly inside the unit ball. Boche showed that if $\pi_{n}$ is monic, the conditions

$$
\sup _{n \geq 1} \sum_{j=1}^{n} \frac{1}{\left|\pi_{n}^{\prime}\right|\left(z_{j n}\right)}<\infty
$$

and for each $\rho \in(0,1)$,

$$
\sup _{n \geq 1} \sup _{|z| \leq \rho}\left|\pi_{n}(z)\right|<\infty
$$

are necessary and sufficient for this type of convergence. These are implied by (4), and so are more general. Of course this is to be expected as the conclusion of [2] does not involve convergence on the unit circle, it involves a consequence of Theorems 3 .

The implication (I) $\Rightarrow(\mathrm{II})$ in Theorem 3 is a little deeper than that in Theorems 1 or 2 - because of the nature of the space $A(\bar{D})$, we have to use the Carleson-Rudin Theorem. This ensures the existence of functions in $A(\bar{D})$ with given boundary values on a closed set of measure 0 on $\Gamma$, and with bounded norm.

We prove Theorems 2 and 3 and the Proposition in Section 2. 


\section{The Proof of Theorem 1}

Recall that given measurable $g: K \rightarrow \mathbb{C}$, its distribution function is

$$
m_{g}(\lambda):=\operatorname{meas}(\{x \in K:|g(x)|>\lambda\}), \lambda \geq 0 .
$$

Here meas denotes linear Lebesgue measure. There is the well known formula $[1$, p.43], [10, p. 172]

$$
\int_{K}|g|^{p}=\int_{0}^{\infty} p t^{p-1} m_{g}(t) d t, p>0 .
$$

We need a consequence of a lemma of Loomis. See [3] or [1] for a discussion of lemmas of this type.

\section{LEMMA 2.1}

Let $c_{1}, c_{2}, \ldots c_{n} \in \mathbb{C}$, and $\theta_{1}, \theta_{2}, \ldots \theta_{n} \in[-\pi, \pi]$. Then for $\lambda>0$,

$$
\text { meas }\left\{\theta \in\left[0, \frac{\pi}{2}\right]:\left|\sum_{j=1}^{n} \frac{c_{j}}{\sin \left(\frac{\theta-\theta_{j}}{2}\right)}\right|>\lambda\right\} \leq \frac{128}{\lambda} \sum_{j=1}^{n}\left|c_{j}\right| .
$$

\section{PROOF}

Let

$$
B:=\sum_{j=1}^{n}\left|c_{j}\right|
$$

Loomis' Lemma asserts that if $\alpha_{1}, \alpha_{2}, \ldots \alpha_{n}>0$ and $t_{1}, t_{2}, \ldots t_{n} \in \mathbb{R}$,

$$
\text { meas }\left\{t \in \mathbb{R}:\left|\sum_{j=1}^{n} \frac{\alpha_{j}}{t-t_{j}}\right|>\lambda\right\}=\frac{2}{\lambda} \sum_{j=1}^{n} \alpha_{j} .
$$

See $[1$, p. 128, Lemma 4.4]. By considering positive and negative parts, and then real and imaginary parts, it is easy to deduce the following consequence: if $d_{1}, d_{2} \ldots d_{n} \in \mathbb{C}$ and $t_{1}, t_{2} \ldots t_{n} \in \mathbb{R}$, then

$$
\text { meas }\left\{t \in \mathbb{R}:\left|\sum_{j=1}^{n} \frac{d_{j}}{t-t_{j}}\right|>\lambda\right\} \leq \frac{32}{\lambda} \sum_{j=1}^{n}\left|d_{j}\right| .
$$

To apply this to the sum in (6), we use the inequality,

$$
\left|\frac{1}{\sin u}-\frac{1}{u}\right| \leq 1,0<|u| \leq \frac{3 \pi}{4} .
$$

Then $\theta_{j} \in[-\pi, \pi], \theta \in\left[0, \frac{\pi}{2}\right]$ imply that

$$
-\frac{\pi}{2} \leq \frac{\theta-\theta_{j}}{2} \leq \frac{3 \pi}{4}
$$

so that if $\theta \neq \theta_{j}$,

$$
\left|\frac{1}{\sin \frac{\theta-\theta_{j}}{2}}-\frac{2}{\theta-\theta_{j}}\right| \leq 1
$$

Hence we obtain,

$$
\left|\sum_{j=1}^{n} \frac{c_{j}}{\sin \left(\frac{\theta-\theta_{j}}{2}\right)}-\sum_{j=1}^{n} \frac{2 c_{j}}{\theta-\theta_{j}}\right| \leq \sum_{j=1}^{n}\left|c_{j}\right|=B .
$$


So, for $\lambda \geq 2 B$,

$$
\begin{aligned}
& \text { meas }\left\{\theta \in\left[0, \frac{\pi}{2}\right]:\left|\sum_{j=1}^{n} \frac{c_{j}}{\sin \left(\frac{\theta-\theta_{j}}{2}\right)}\right|>\lambda\right\} \\
\leq & \operatorname{meas}\left\{\theta \in\left[0, \frac{\pi}{2}\right]:\left|\sum_{j=1}^{n} \frac{2 c_{j}}{\theta-\theta_{j}}\right|>\lambda-B\right\} \\
\leq & \frac{32}{\lambda-B} \sum_{j=1}^{n}\left(2\left|c_{j}\right|\right) \leq \frac{128}{\lambda} B,
\end{aligned}
$$

by (7). Also for $\lambda<2 B$, we have the trivial bound

$$
\begin{aligned}
& \quad \text { meas }\left\{\theta \in\left[0, \frac{\pi}{2}\right]:\left|\sum_{j=1}^{n} \frac{c_{j}}{\sin \left(\frac{\theta-\theta_{j}}{2}\right)}\right|>\lambda\right\} \\
& \leq \frac{\pi}{2} \leq \frac{128}{\lambda} B .
\end{aligned}
$$

THE PROOF OF THEOREM $2,($ II $) \Rightarrow($ I $)$

Let us assume initially that

$$
\|f\|_{L_{\infty}(K)} \leq 1
$$

Now we can write

$$
\Upsilon_{n}[f](\theta)=\omega_{n}(\theta) \sum_{j=0}^{2 n} \frac{f\left(\theta_{j n}\right)}{\omega_{n}^{\prime}\left(\theta_{j n}\right)\left(2 \sin \left(\frac{\theta-\theta_{j n}}{2}\right)\right)}=: \omega_{n}(\theta) g_{n}(\theta) .
$$

Let $p>0$. Then

$$
\left\|\Upsilon_{n}[f] v\right\|_{L_{p}(K)} \leq\left\|\omega_{n} v\right\|_{L_{2 p}(K)}\left\|g_{n}\right\|_{L_{2 p}(K)} .
$$

To estimate the norm of $g_{n}$, we first consider the range $\left[0, \frac{\pi}{2}\right]$ and use its distribution function

$$
m_{g_{n}}(\lambda):=\text { meas }\left\{\theta \in\left[0, \frac{\pi}{2}\right] \cap K:\left|g_{n}(\theta)\right|>\lambda\right\}, \lambda>0
$$

By the lemma above,

$$
m_{g_{n}}(\lambda) \leq \frac{128}{\lambda} \sum_{j=0}^{2 n}\left|\frac{f}{2 \omega_{n}^{\prime}}\left(\theta_{j n}\right)\right| \leq \frac{64}{\lambda} \sum_{j=0}^{2 n} \frac{1}{\left|\omega_{n}^{\prime}\left(\theta_{j n}\right)\right|}=: \frac{64}{\lambda} \Omega_{n}, \lambda>0 .
$$

Moreover, there is the trivial bound $m_{g_{n}}(\lambda) \leq \frac{\pi}{2}$. We now use (5):

$$
\begin{gathered}
\left\|g_{n}\right\|_{L_{2 p}\left(\left[0, \frac{\pi}{2}\right] \cap K\right)}^{2 p}=2 p \int_{0}^{\infty} \lambda^{2 p-1} m_{g_{n}}(\lambda) d \lambda \\
\leq 2 p \int_{0}^{\infty} \lambda^{2 p-1} \min \left\{\frac{\pi}{2}, \frac{64 \Omega_{n}}{\lambda}\right\} d \lambda=2 p \Omega_{n}^{2 p} \int_{0}^{\infty} s^{2 p-1} \min \left\{\frac{\pi}{2}, \frac{64}{s}\right\} d s=: C_{p}^{2 p} \Omega_{n}^{2 p} .
\end{gathered}
$$

Of course $C_{p}$ is finite if $p<\frac{1}{2}$. By translations of the intervals $\left[\frac{\pi}{2}, \pi\right],\left[\pi, \frac{3 \pi}{2}\right]$ and $\left[\frac{3 \pi}{2}, 2 \pi\right]$, we see that similar estimates hold over those intervals, and hence,

$$
\left\|g_{n}\right\|_{L_{2 p}(K)}^{2 p} \leq 4 C_{p}^{2 p} \Omega_{n}^{2 p} .
$$


Then (8) gives

$$
\sup _{n}\left\|\Upsilon_{n}[f] v\right\|_{L_{p}(K)} \leq 4^{1 /(2 p)} C_{p} \sup _{n}\left\|\omega_{n} v\right\|_{L_{2 p}(K)} \Omega_{n}=: A<\infty
$$

by (2), provided $2 p \leq r$. For general $f \in \mathcal{C}(K)$, without assuming $\|f\|_{L_{\infty}(K)} \leq 1$, we deduce that

$$
\sup _{n}\left\|\Upsilon_{n}[f] v\right\|_{L_{p}(K)} \leq A\|f\|_{L_{\infty}(K)}
$$

Then if $S$ is a trigonometric polynomial of degree $\leq \ell$ and $n \geq \ell$,

$$
\begin{aligned}
& \left\|\quad\left(f-\Upsilon_{n}[f]\right) v\right\|_{L_{p}(K)}^{p} \leq\|(f-S) v\|_{L_{p}(K)}^{p}+\left\|\Upsilon_{n}[S-f] v\right\|_{L_{p}(K)}^{p} \\
& \leq \quad\|f-S\|_{L_{\infty}(K)}^{p}\|v\|_{L_{p}(K)}^{p}+A^{p}\|S-f\|_{L_{\infty}(K)}^{p} .
\end{aligned}
$$

Now by Tietze's extension theorem, each function continuous on $K$ has an extension to a function continuous on $[0,2 \pi]$. Also our hypothesis that $f(0)=f(2 \pi)$ if both 0 and $2 \pi$ lie in $K$ shows that we may ensure that $f$ is $2 \pi$-periodic. Then Weierstrass' Theorem for trigonometric polynomials ensures that we can find a trigonometric polynomial $S$ for which $\|f-S\|_{L_{\infty}(K)}$ is as small as we please.

We proceed with the converse. The basic idea appeared in a paper of Ying Guang Shi [11, pp. 30-31, Lemma 1], although the technicalities are a little more complicated in the trigonometric case:

\section{THE PROOF OF THEOREM 2 (I) $\Rightarrow$ (II)}

Assume that we have the convergence (1). We may assume that $p \leq q$. (For if (1) holds for a given $p$, then it holds for smaller $p$ ). Then the uniform boundedness principle gives

$$
\left\|\left(f-\Upsilon_{n}[f]\right) v\right\|_{L_{p}(K)} \leq C\|f\|_{L_{\infty}(K)}
$$

where $C$ is independent of $n$ and $f$, and consequently, for some possibly different $C$,

$$
\left\|\Upsilon_{n}[f] v\right\|_{L_{p}(K)} \leq C\left(\|f\|_{L_{\infty}(K)}+\|f v\|_{L_{p}(K)}\right) .
$$

Of course if $p<1$, the space

$$
\left\{h: K \rightarrow \mathbb{R}:\|h v\|_{L_{p}(K)}<\infty\right\}
$$

is not a normed space, but it is a topological vector space, while $\mathcal{C}(K)$ is a Banach space, and there is a version of the uniform boundedness principle that may be applied. See, for example, [9, p. 44, Thm. 2.6]. Next, fix $n \geq 1$ and choose $f$ continuous on $K$ such that

$$
f\left(\theta_{k n}\right)=\frac{\omega_{n}^{\prime}\left(\theta_{k n}\right)}{\left|\omega_{n}^{\prime}\left(\theta_{k n}\right)\right|}, \quad 0 \leq k \leq 2 n
$$

and $\|f\|_{L_{\infty}(K)}=1$ (for example, we could choose $f$ to be a piecewise linear function). Let

$$
S_{n}(\theta):=\omega_{n}(\theta) \sum_{k=0}^{2 n} \frac{1}{\left|\omega_{n}^{\prime}\left(\theta_{k n}\right)\right|}
$$


We see that

$$
\begin{aligned}
S_{n}(\theta) & =\omega_{n}(\theta) \sum_{k=0}^{2 n} \frac{f\left(\theta_{k n}\right)}{\omega_{n}^{\prime}\left(\theta_{k n}\right)}=\sum_{k=0}^{2 n} f\left(\theta_{k n}\right)\left(2 \sin \frac{\theta-\theta_{k n}}{2}\right) \tau_{k n}(\theta) \\
& =\left[2 \sin \frac{\theta}{2}\right] \Upsilon_{n}[g](\theta)-\left[2 \cos \frac{\theta}{2}\right] \Upsilon_{n}[h](\theta),
\end{aligned}
$$

where

$$
g(\theta):=f(\theta) \cos \frac{\theta}{2} ; h(\theta):=f(\theta) \sin \frac{\theta}{2} .
$$

Then (10) and the fact that $|g| \leq|f| \leq 1$ and $|h| \leq|f| \leq 1$ give

$$
\begin{aligned}
\left\|S_{n} v\right\|_{L_{p}(K)} & \leq 2^{1+1 / p}\left(\left\|\Upsilon_{n}[g] v\right\|_{L_{p}(K)}+\left\|\Upsilon_{n}[h] v\right\|_{L_{p}(K)}\right) \\
& \leq 2^{1+1 / p} C\left(\|g\|_{L_{\infty}(K)}+\|g v\|_{L_{p}(K)}+\|h\|_{L_{\infty}(K)}+\|h v\|_{L_{p}(K)}\right) \\
& \leq 2^{2+1 / p} C\left(1+\|v\|_{L_{p}(K)}\right)=: C_{1} .
\end{aligned}
$$

As $C_{1}$ is finite (recall $p \leq q$ ) and independent of $n$, we have (2) with $r=p$.

We turn to

\section{PROOF OF THE PROPOSITION}

Recall that if $g \in H_{p}$, and $g^{*}$ denotes its non-tangential boundary values, while $\Gamma$ denotes the unit circle, then

$$
\|g\|_{H_{p}}=\left(\frac{1}{2 \pi} \int_{0}^{2 \pi}\left|g^{*}\left(e^{i \theta}\right)\right|^{p} d \theta\right)^{1 / p}=:\|g\|_{L_{p}(\Gamma)} .
$$

Now assume that $f \in L_{p}(\Gamma)$ and that

$$
\lim _{n \rightarrow \infty}\left\|f-L_{n}[f]\right\|_{L_{p}(\Gamma)}=0 .
$$

We see that $\left\{L_{n}[f]\right\}_{n=1}^{\infty}$ is a Cauchy sequence in $H_{p}$. Indeed, the assumed convergence gives

$$
\left\|L_{n}[f]-L_{m}[f]\right\|_{H_{p}}=\left\|L_{n}[f]-L_{m}[f]\right\|_{L_{p}(\Gamma)} \rightarrow 0,
$$

as $m, n \rightarrow \infty$. Since $H_{p}$ is complete (even if $p<1$ ), there exists $F \in H_{p}$ such that

$$
\left\|L_{n}[f]-F\right\|_{H_{p}} \rightarrow 0, n \rightarrow \infty .
$$

But then as $n \rightarrow \infty$,

$$
\left\|L_{n}[f]-F^{*}\right\|_{L_{p}(\Gamma)}=\left\|L_{n}[f]-F\right\|_{H_{p} \rightarrow 0,}
$$

and so $f=F^{*}$.

THE PROOF OF THEOREM 3 (II) $\Rightarrow(\mathrm{I})$

Since this is similar to that of Theorem (II) $\Rightarrow(\mathrm{I})$, we present a brief outline. Write 
ON MEAN CONVERGENCE OF TRIGONOMETRIC INTERPOLANTS, AND THEIR UNIT CIRCLE ANALOGUES, FOR GENERA

$z=e^{i \theta}$ and $z_{j n}=e^{i \theta_{j n}}$ for all $j, n$. We can write

$$
\begin{aligned}
L_{n}[f](z) & =\pi_{n}(z) \sum_{j=1}^{n} \frac{f\left(z_{j n}\right)}{\pi_{n}^{\prime}\left(z_{j n}\right)\left(z-z_{j n}\right)} \\
& =\frac{\pi_{n}(z)}{2 i e^{i \theta / 2}} \sum_{j=1}^{n} \frac{f\left(z_{j n}\right)}{\pi_{n}^{\prime}\left(z_{j n}\right) e^{i \theta_{j n} / 2}\left(\sin \left(\frac{\theta-\theta_{j n}}{2}\right)\right)} .
\end{aligned}
$$

We can now follow the same steps as for $\Upsilon_{n}[\cdot](\theta)$ to prove boundedness of $\left\{L_{n}\right\}_{n=1}^{\infty}$. Note that of course

$$
\left\|L_{n}[f]\right\|_{H_{p}}^{p}=\frac{1}{2 \pi} \int_{0}^{2 \pi}\left|L_{n}[f]\left(e^{i \theta}\right)\right|^{p} d \theta .
$$

The convergence in $A(\bar{D})$ follows as the algebraic polynomials are dense in that space.

\section{THE PROOF OF THEOREM 3 (I) $\Rightarrow$ (II)}

This is similar to the proof of Theorem 2 (I) $\Rightarrow$ (II), but because of the need to apply the Carleson-Rudin theorem, we give the details. As in the analogous part of Theorem 2, the uniform boundedness principle applied to the topological vector space $H_{p}$ and the Banach space $A(\bar{D})$ shows that there exists $C_{1}>0$, independent of $f$ and $n$, such that for $f \in A(\bar{D})$,

$$
\begin{array}{ll} 
& \left\|L_{n}[f]\right\|_{L_{p}(\Gamma)}=\left\|L_{n}[f]\right\|_{H_{p}} \\
\leq & C_{1}\left(\|f\|_{L_{\infty}(\Gamma)}+\|f\|_{H_{p}}\right) \\
\leq & 2 C_{1}\|f\|_{L_{\infty}(\Gamma)} .
\end{array}
$$

Now fix $n \geq 1$. Since $\left\{z_{j n}\right\}_{j=1}^{n}$ is a discrete set, and so any function defined on it is trivially continuous, the Carleson-Rudin Theorem [4, pp. 125-6] ensures the existence of $f \in A(\bar{D})$ with

$$
f\left(z_{j n}\right)=e^{i \arg \pi_{n}^{\prime}\left(z_{j n}\right)}, 1 \leq j \leq n
$$

and $\|f\|_{L_{\infty}(\Gamma)}=1$. Then

$$
\begin{aligned}
S_{n}(z) & :=\pi_{n}(z) \sum_{j=1}^{n} \frac{1}{\left|\pi_{n}^{\prime}\left(z_{j n}\right)\right|} \\
& =\pi_{n}(z) \sum_{j=1}^{n} \frac{f\left(z_{j n}\right)}{\pi_{n}^{\prime}\left(z_{j n}\right)} \\
& =\sum_{j=1}^{n} f\left(z_{j n}\right)\left(z-z_{j n}\right) \ell_{j n}(z) \\
& =z L_{n}[f](z)-L_{n}[g](z),
\end{aligned}
$$

where $g(z):=z f(z)$. Then

$$
\left\|S_{n}\right\|_{H_{p}} \leq 2^{1 / p}\left(\left\|L_{n}[f]\right\|_{H_{p}}+\left\|L_{n}[g]\right\|_{H_{p}}\right) \leq 2^{2+1 / p} C_{1},
$$

by (11).

\section{Remark}


The obvious way to define $f$ above is to choose $f$ to satisfy (12), and then to define $f$ in such a way that $\arg f$ is a piecewise linear function. Then $f \in C(\Gamma)$ and $|f|=1$ on $\Gamma$. But such an $f$ is not obviously the restriction to $\Gamma$ of a function in $A(\bar{D})$, and for some arrays, will not be. Nor is there any obvious construction, for example, involving Blaschke products, that will do the job. That is why we need a relatively deep result like the Carleson-Rudin theorem.

\section{REFERENCES}

[1] Bennett, C., and Sharpley, R.: Interpolation of Operators, Academic Press, New York, 1988.

[2] Boche, H.: Konvergente Lagrange-Interpolationspolynome für die Disk-Algebra, Numerische Mathematik, 88, 1-7(2001).

[3] Borwein, P. and Erdelyi, T.: Polynomials and Polynomial Inequalities, Springer, New York, 1995.

[4] Garnett, J.B.: Bounded Analytic Functions, Academic Press, Orlando, 1981.

[5] Lubinsky, D.S.: On Mean Convergence of Lagrange Interpolation for General Arrays, J. Approx. Theory, 104, 220-225(2001).

[6] Lubinsky, D.S.: A Taste of Erdös on Interpolation, to appear in Proceedings of conference in memory of P. Erdös, Colloq. Math. Soc. Janos Bolyai.

[7] Lubinsky, D.S.: On Weighted Mean Convergence of Lagrange Interpolation for General Arrays, manuscript.

[8] Mastroianni, G.: Boundedness of the Lagrange Operator in Some Functional Spaces. A Survey, (in) Approximation Theory and Function Spaces, Bolyai Math. Soc. Studies, 5, 117139(1996).

[9] Rudin, W.: Functional Analysis, Tata McGraw Hill, New Delhi, 1973.

[10] Rudin, W.: Real and Complex Analysis, 3rd edition, McGraw Hill, Singapore, 1987.

[11] Shi, Y.G.: Mean Convergence of Interpolatory Processes on an Arbitrary System of Nodes, Acta Math. Hungar., 70, 27-38(1996).

[12] Szabados, J. and Vertesi, P.: Interpolation of Functions, World Scientific, Singapore, 1990.

[13] Szabados, J. and Vertesi, P.: A Survey on Mean Convergence of Interpolatory Processes, J. Comp. Appl. Math., 43, 3-18(1992).

[14] Timan, A.F.: Theory of Approximation of Functions of a Real Variable, Dover, New York, 1994.

[15] Zygmund, A.: Trigonometric Series, Vols. I-II, Cambridge University Press, Cambridge, 1990.

School of Mathematics, Georgia Institute of Technology, Atlanta, Georgia Ga 30332-0160, USA, and, The John Knopfmacher Centre, School of Mathematics, Witwatersrand University, Wits 2050, South Africa, E-Mail: Lubinsky@math.gatech.edu 